Article

\title{
Sequential Photodynamic Therapy with Phthalocyanine Encapsulated Chitosan-Tripolyphosphate Nanoparticles and Flucytosine Treatment against Candida tropicalis
}

\author{
Yi-Hsuan Hsieh ${ }^{1}$, Wen-Ching Chuang ${ }^{2}$, Kun-Hua Yu ${ }^{2}$, Cheng-Ping Jheng ${ }^{2}$ and \\ Cheng-I Lee $2,3,4, *$ (D) \\ 1 Department of Clinical Pathology, Dalin Tzu Chi Hospital, Buddhist Tzu Chi Medical Foundation, \\ Chia-Yi 62247, Taiwan; dm989587@tzuchi.com.tw \\ 2 Department of Biomedical Sciences, National Chung Cheng University, Min-Hsiung Chia-Yi 62102, Taiwan; \\ danny7910791@gmail.com (W.-C.C.); ykhuna@gmail.com (K.-H.Y.); \\ chengpingkevincheng@hotmail.com (C.-P.J.) \\ 3 Center for Innovative Research on Aging Society (CIRAS), National Chung Cheng University, \\ Min-Hsiung Chia-Yi 62102, Taiwan \\ 4 Center for Nano Bio-detections, Advanced Institute of Manufacturing with High-tech Innovations (AIM-HI), \\ National Chung Cheng University, Min-Hsiung Chia-Yi 62102, Taiwan \\ * Correspondence: biocil@ccu.edu.tw; Tel.: +886-5-2729-157
}

Received: 14 November 2018; Accepted: 27 December 2018; Published: 4 January 2019

\begin{abstract}
Antibiotic resistance has become a crisis. Candida tropicalis (C. tropicalis) is one of the most highly virulent and drug-resistant pathogens. An alternative antimicrobial therapy to eradicate C. tropicalis effectively, without the risk of developing drug-resistance, is needed. Photodynamic therapy (PDT) is an alternative therapy that does not carry the risk of undesired drug resistance. To target the pathogens and to enhance the cellular penetration of the applied photosensitizer, we fabricated cationic chitosan/tripolyphosphate nanoparticles to encapsulate phthalocyanine. Our strategy promotes the uptake of phthalocyanine four-fold. This enhanced PDT can effectively inhibit planktonic $C$. tropicalis, such that only $\sim 20 \%$ of $C$. tropicalis in the test survived; but it has a limited ability to inhibit adherent $C$. tropicalis. Further tests with adherent $C$. tropicalis indicated that sequential treatment with PDT and flucytosine significantly eliminates pseudohyphae and yeast-like $C$. tropicalis cells. The cell viability is only $\sim 10 \%$ after this sequential treatment. This study provides evidence of an effective therapy against drug resistant $C$. tropicalis, and this strategy can be potentially applied to other pathogens.
\end{abstract}

Keywords: photodynamic therapy; Candida tropicalis; chitosan; phthalocianine; flucytosine

\section{Introduction}

Antibiotic resistance has become a crisis [1]. Candidiasis is one of the most frequent fungal infections [2]. In the intensive care unit of medical centers, infections with Candida species (Candida spp.) have become very common [3]. Candidiasis causes high mortality in patients with immunodeficiency [4]. Candida albicans (C. albicans) is the most commonly encountered human fungal pathogen [5]. In recent years, the overuse of antifungal drugs has led to resistance in many non-albicans Candida spp., such as Candida tropicalis (C. tropicalis) and Candida glabrata (C. glabrata) [6,7]. In addition, the resistance of these species to azole drugs results in serious infections [8,9]. C. tropicalis is an extremely common pathogen that causes human disease in tropical countries [10]. The virulence of 
C. tropicalis can be more severe than that of C. albicans in the human intestine, particularly in oncology patients [11,12]. The incidence of urinary tract infections with $C$. tropicalis is second to that with C. albicans in some Asian countries [13,14].

In the treatment of candidiasis, the drugs that are commonly used to treat Candida infections include amphotericin B, fluconazole, liposomal amphotericin B, and flucytosine $[15,16]$. The antifungal mechanisms of these antifungal agents are different [17]. Amphotericin B interacts with ergosterol to disrupt fungal cell membrane by pore formation. Fluconazole destabilizes the membrane structure through reducing the synthesis of ergosterol and incorporating the precursor of sterol into the cell membrane. Flucytosine interferes with the synthesis of fungal proteins and DNA. All these antifungal agents have some side effects [17]. With the use of these chemical antifungal agents, the recurrence of infection after treatment is very common [18], mainly due to the development of resistance [19,20]. Similar to C. albicans, C. tropicalis is a polymorphic fungus that can grow either as ovoid-shaped budding yeast or as parallel-walled true hyphae. As elongated ellipsoid cells, pseudohyphae develop during the transition between budding and hyphal growth, and this transition is essential for virulence [21]. Biofilms consist of pseudohyphae, and hyphae are notorious for intrinsic resistance to conventional antifungal therapeutics [8].

Photodynamic therapy is a promising alternative treatment in cancers [22] and microbial diseases $[23,24]$. The combination of chemical treatment and photodynamic therapy (PDT) greatly enhances antimicrobial efficiency in C. albicans [25]. Many photosensitizers have been approved by the Food and Drug Administration in the US, including Photofrin, Levulan, Metvix, and Visudyne [26]. Compared with traditional therapies involving antimicrobial medicines, PDT has the advantages of localized application, low risk of side effects, and nonexistent drug-resistance [27]. However, the use of PDT in microbial infections is limited in comparison to its use for cancer treatment. Considering the significant increase of drug-resistant Candida infections, it is important to develop antimicrobial PDT as an alternative therapy to eradicate pathogenic microorganisms effectively, without risking drug-resistance.

Photodynamic therapy is a form of phototherapy involving three main components-light, a photosensitizer, and oxygen molecules. The photosensitizer is excited from the ground singlet state to the excited singlet state when it receives light energy at a specific wavelength. The photosensitizer in the excited singlet state crosses to the first triplet state. When returning to the ground state, the photosensitizer releases some energy to a nearby triplet molecule oxygen $\left({ }^{3} \mathrm{O}_{2}\right)$, forming the excited state singlet oxygen $\left({ }^{1} \mathrm{O}_{2}\right)$ in a type II reaction. In the type I reaction, the excited photosensitizer reacts with the biomolecule and transfers the energy through electron transfer, producing free radicals. Further reaction between singlet oxygen and free radicals leads to the formation of a reactive oxygen species. The photosensitizer is a critical mediator in PDT. However, the solubility of photosensitizers is usually low. Furthermore, tumor-targeted forms of PDT have been developed [28,29]. The development of PDT targeted to pathogens is in progress [30], but it is not as mature yet as tumor-targeted PDT. The poor solubility and selectivity limit the applications of PDT. Nanotechnology has been applied to PDT to overcome the poor water-solubility of photosensitizers and to enhance their efficiency [31]. Encapsulation of hydrophobic photosensitizers with hydrophilic carriers increases the efficiency of drug delivery and systemic administration. Chitosan, a polysaccharide produced from the deacetylation of chitin, has been widely used as a delivery particle due to its desirable properties, which include biocompatibility, biodegradability, and lack of toxicity [32]. Therefore, chitosan-based materials have been used in PDT [33-35]. Fabrication of chitosan-based nanoparticles can be carried out by an intermolecular ionic cross-linking between protonated chitosan and anionic phosphate groups, such as tripolyphosphate (TPP) [34,36]. In addition, chitosan/TPP nanoparticles carrying the desired charge can be fabricated by adjusting the ratio of the chitosan and TPP.

Phthalocyanine derivatives, which have similar structures to porphyrin, have been used as photosensitizers $[26,37]$. Similar to the situation with other photosensitizers, their low solubility and poor cell penetrability limits their application to PDT. To overcome these limitations, we used 
a nanotechnology approach, in which chitosan/TPP nanoparticles (NP) were the carrier of phthalocyanine to develop an efficient antimicrobial PDT against $C$. tropicalis. The physical, chemical, and cellular properties of phthalocyanine encapsulated chitosan/TPP nanoparticles (FNP) were characterized. The antimicrobial effect of PDT using FNP was evaluated in both planktonic and adherent cultures of $C$. tropicalis. Antimicrobial activity in the combination treatment of FNP-PDT and flucytosine against $C$. tropicalis was evaluated.

\section{Materials and Methods}

\subsection{Preparation of Chitosan and Tripolyphosphate}

Chitosan (Sigma-Aldrich, St. Louis, MO, US) was added to $100 \mathrm{~mL}$ of acetic acid at 2\% (v/v) to reach a concentration of $2 \%(w / v)$, and stirred at room temperature overnight. After the chitosan was completely dissolved, the $\mathrm{pH}$ of the solution was adjusted to 6, and the solution was then filtered with a $0.22 \mu \mathrm{m}$ membrane. Sodium tripolyphosphate (Sigma-Aldrich, St. Louis, MO, USA) was dissolved in $\mathrm{H}_{2} \mathrm{O}$ to reach a concentration of $1 \%(w / v)$, and then filtered with a $0.22 \mu \mathrm{m}$ membrane. In the subsequent experiment, the chitosan and TPP solutions were diluted with $\mathrm{H}_{2} \mathrm{O}$ to the desired concentration.

\subsection{Fabrication of FNP}

To generate FNP, the photosensitizer was mixed with $0.1 \%$ TPP $(w / v)$ prior to cross-linking. The ionic cross-linking reaction was initiated by the slow addition of chitosan at $0.5 \%(w / v)$ under probe-type ultrasonication on ice. The photosensitizer used in this work is iron(III) phthalocyanine- $4,4^{\prime}, 4^{\prime \prime}, 4^{\prime \prime \prime}$-tetrasulfonic acid (FePC). The fabrication procedure of FNP is illustrated in Figure 1. The concentration of FNP was determined by the content of encapsulated FePC.

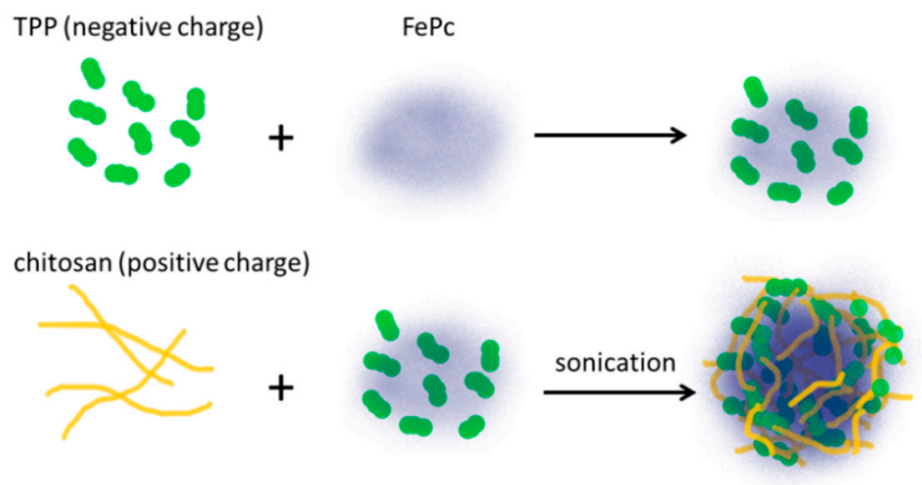

Figure 1. A schematic diagram for the fabrication of phthalocyanine encapsulated chitosan/TPP nanoparticles (FNP) from chitosan, tripolyphosphate (TPP), and phthalocyanine- $4,4^{\prime}, 4^{\prime \prime}, 4^{\prime \prime \prime}$-tetrasulfonic acid (FePC).

\subsection{Characterization of Nanoparticles}

The fabricated nanoparticles were lyophilized and the powdered samples were placed on $\mathrm{KBr}$ to form pellets under compression for FT-IR measurement. The FT-IR spectra were recorded from $4000 \mathrm{~cm}^{-1}$ to $700 \mathrm{~cm}^{-1}$ at a resolution of $4 \mathrm{~cm}^{-1}$ with an FT-IR spectrometer (VERTEX 70v, Bruker, Billerica, MA, US) equipped with a mercury cadmium telluride detector.

The physical properties, including hydrodynamic diameter $\left(\mathrm{D}_{\mathrm{h}}\right)$, polydispersity indexes (PDI) and zeta-potential of fabricated nanoparticles, were measured by dynamic light scattering (DLS, Nano-ZS90, Malvern, Worcestershire, UK). 


\subsection{Determining the Encapsulation Efficiency of Chitosan/TPP Nanoparticles}

Phthalocyanine-encapsulated nanoparticles were separated from the aqueous suspension containing FePC (Sigma-Aldrich, St. Louis, MO, US) by centrifugation at 12,000 rpm for $5 \mathrm{~min}$. The supernatant was collected and the free FePC was determined by measuring the absorbance at $630 \mathrm{~nm}$ using UV-VIS spectroscopy (S-3100, Scinco, Seoul, Korea). The encapsulation efficiency of FePC in nanoparticles was calculated as follows: $[(A-B) / A] \times 100$, where $A$ is the total amount of FePC and B is the amount of free FePC.

\subsection{Determination of Release Efficiency of Phthalocyanine from Chitosan/TPP Nanoparticles}

Release of FePC from nanoparticles was measured in phosphate buffer solution (PBS, Avantor, Center Valley, PA, USA) at different time intervals. Primarily, FNP was removed from free FePC by centrifugation at 12,000 rpm for $5 \mathrm{~min}$. The released FePC in the supernatant was quantified by absorbance at $630 \mathrm{~nm}$.

\subsection{Culture of Planktonic C. albicans and C. tropicalis}

In this study of C. tropicalis, C. albicans was used as a comparison. Both C. tropicalis and C. albicans were provided by Dalin Tzu Chi Hospital (Chia-Yi, Taiwan). Suspensions $\left(1.5 \times 10^{8}\right.$ cells $\left./ \mathrm{mL}\right)$ of $C$. albicans (ATCC 90029) or C. tropicalis (ATCC 13803) were cultured in yeast extract-peptone-dextrose (YEPD) growth medium in glass tubes at $37^{\circ} \mathrm{C}$. Antifungal agents or photosensitizers were added to C. albicans or C. tropicalis in YEPD growth medium and incubated at $37^{\circ} \mathrm{C}$. To determine cell viability, aliquots of 1000-fold serial dilutions of each sample were seeded on YEPD agar plates. All plates were aerobically incubated at $37^{\circ} \mathrm{C}$ for $18 \mathrm{~h}$, and the number of colony forming units per milliliter $(\mathrm{CFU} / \mathrm{mL})$ was counted from the plates. The tested fluconazole concentration was lower than or equal to $208 \mu \mathrm{M}(64 \mu \mathrm{g} / \mathrm{mL})$, the minimum inhibitory concentration (MIC) reported by Clinical Laboratory Standards Institute [38]. The tested concentrations of flucytosine were in the range of various MIC values measured from flucytosin-resistant C. tropicalis [39]. Notably, the activity of antifungal agents is suggested to be tested in RPMI 1640 medium [40]. In this work, we kept the Candida species in YEPD medium in all experiments.

\subsection{Culture of Adherent C. tropicalis}

To generate adherent cultures of $C$. tropicalis, $100 \mu \mathrm{L}$ of a C. tropicalis suspension was transferred into a 96-well culture plate at a concentration of $1.5 \times 10^{4}$ cells $/ \mathrm{mL}$ and incubated at $37{ }^{\circ} \mathrm{C}$ in an orbital shaker at $80 \mathrm{rpm}$ overnight. Subsequently, samples were washed with $150 \mu \mathrm{L}$ of PBS to remove suspended cells. YEPD medium containing antifungal agents or photosensitizer was added for further incubation at $37^{\circ} \mathrm{C}$. The incubation with antifungal agents lasted 24 or $48 \mathrm{~h}$, whereas incubation with photosensitizer lasted $4 \mathrm{~h}$.

The MTT cell proliferation assay was used to measure the viability of adherent $C$. tropicalis cells. After the addition of $100 \mu \mathrm{L}$ of medium containing $5 \mu \mathrm{L}$ of MTT, C. tropicalis cells were incubated for $4 \mathrm{~h}$ at $37^{\circ} \mathrm{C}$ in the dark, and formazan absorbance was measured at $595 \mathrm{~nm}$ in an ELISA reader.

A $t$-test was performed for statistical analysis in all experiments using $C$. tropicalis. The reported values are the mean values of three replicates. Statistical significance was indicated at $p<0.05\left(^{*}\right)$, $\left.p<0.01{ }^{(* *}\right)$, or $p<0.001\left({ }^{* * *}\right)$.

\subsection{PDT Experiments}

PDT experiments were carried out by illuminating FePC- or FNP-treated C. tropicalis using a homemade device consisting of an array of 20 red LEDs (peak emission wavelength at $630 \mathrm{~nm}$, $11.0 \pm 1 \mathrm{~mW} / \mathrm{cm}^{2}$ ). After illumination for $30 \mathrm{~min}$, the accumulated photoenergy was $20 \mathrm{~J} / \mathrm{cm}^{2}$. FePC or FNP solutions were added to C. tropicalis suspensions to reach the desired concentration. A $1 \mathrm{~mL}$ mixture of $C$. tropicalis and photosensitizer was transferred to a 96-well plate and incubated in 
the dark for $4 \mathrm{~h}$. Subsequent illumination for $30 \mathrm{~min}$ activated the PDT. In experiments combining PDT with chemical therapy, C. tropicalis was treated with $128 \mu \mathrm{M}$ flucytosine prior to or after the PDT experiments.

\subsection{Cellular Uptake of FePC in FePC- and FNP-Treated C. tropicalis}

Candida tropicalis was cultured in test tubes at a concentration of $1 \times 10^{8}$ cells $/ \mathrm{mL}$ for treatments with $80 \mu \mathrm{M}$ FePC or FNP. After incubation for $4 \mathrm{~h}$, the cells were washed twice with $\mathrm{H}_{2} \mathrm{O}$ and spun down at 13,000 rpm for $1 \mathrm{~min}$. The cell pellet mixed with $\mathrm{H}_{2} \mathrm{O}$ was sonicated. Subsequently, the cell lysate was centrifuged at 13,000 rpm for $5 \mathrm{~min}$. The concentration of FePC in the suspension was quantified by measuring the absorbance at $630 \mathrm{~nm}$.

\section{Results}

\subsection{Fabrication and Characterization of Nanoparticles}

The chitosan/TPP nanoparticles were fabricated based on previously reported conditions [34]. The $\mathrm{D}_{\mathrm{h}}$, PDI, and zeta potential of FNP measured by DLS were $325.2 \pm 23.3 \mathrm{~nm}, 0.427 \pm 0.094$, and $26.4 \pm 3.2 \mathrm{mV}$, respectively. The fabricated FNP was very stable as tested in time-course DLS measurements shown in Figure $2 \mathrm{a}$, and both $\mathrm{D}_{\mathrm{h}}$ and PDI were close to the initial values. The encapsulation efficiency of FePC in FNP was $80.0 \pm 5.0 \%$. In $4 \mathrm{~h}, 80 \%$ of the FePC was released from FNP into the aqueous solution, as shown in Figure 2b. We quantified FePC in C. tropicalis after treatment with FePC or FNP. As shown in Figure 2c, the uptake of FePC was very limited. Significantly, the uptake of FePC was enhanced four-fold when treated with FNP.

(a)

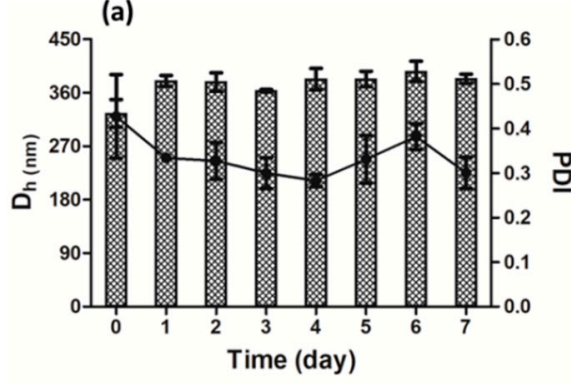

(b)

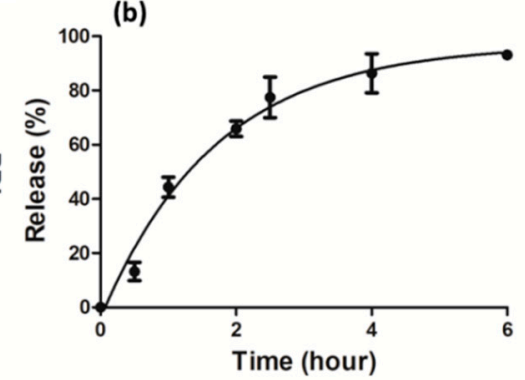

(c)

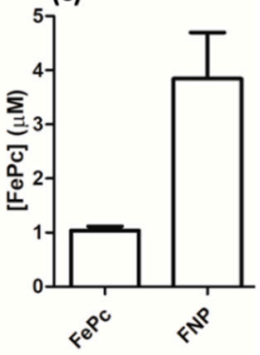

Figure 2. Characterization of FNP. (a) Time-course measurements of $D_{h}$ and polydispersity indexes (PDI) by dynamic light scattering (DLS) shown in columns and dots, respectively; (b) the release of FePC from FNP; (c) uptake of FePC in C. tropicalis after treatment with $80 \mu \mathrm{M} \mathrm{FePC}$ or FNP for $4 \mathrm{~h}$.

\subsection{Spectroscopic Characterization of Nanoparticles}

The composition of nanoparticles was confirmed by FT-IR for the vibrational features of the functional groups. As shown in Figure 3a, the FT-IR spectrum of chitosan reveals stretches of $\mathrm{O}-\mathrm{H}$ and $\mathrm{N}-\mathrm{H}$ at $3200-3600 \mathrm{~cm}^{-1}, \mathrm{C}=\mathrm{O}$ at $1661 \mathrm{~cm}^{-1}, \mathrm{C}-\mathrm{O}$ at $1076 \mathrm{~cm}^{-1}$, and $\mathrm{C}-\mathrm{H}\left(\mathrm{sp}^{3}\right)$ at $2800-3000 \mathrm{~cm}^{-1}$. TPP can be recognized by FT-IR bands at 1165 and $896 \mathrm{~cm}^{-1}$ for $\mathrm{P}=\mathrm{O}$ and $\mathrm{P}-\mathrm{O}-\mathrm{P}$ stretching, respectively, as shown in Figure 3b. The FT-IR spectrum of FePC illustrated in Figure $3 \mathrm{c}$ includes $\mathrm{C}=\mathrm{N}$ stretching of pyrrole at $1463 \mathrm{~cm}^{-1}, \mathrm{Fe}-\mathrm{N}$ of pyrrole at $1105 \mathrm{~cm}^{-1}$ and $-\mathrm{SO}_{3}$ stretching at $1033 \mathrm{~cm}^{-1}$ [41]. In FNP, vibrational bands representing chitosan, TPP, and FePC are all present, as shown in Figure 3d. 


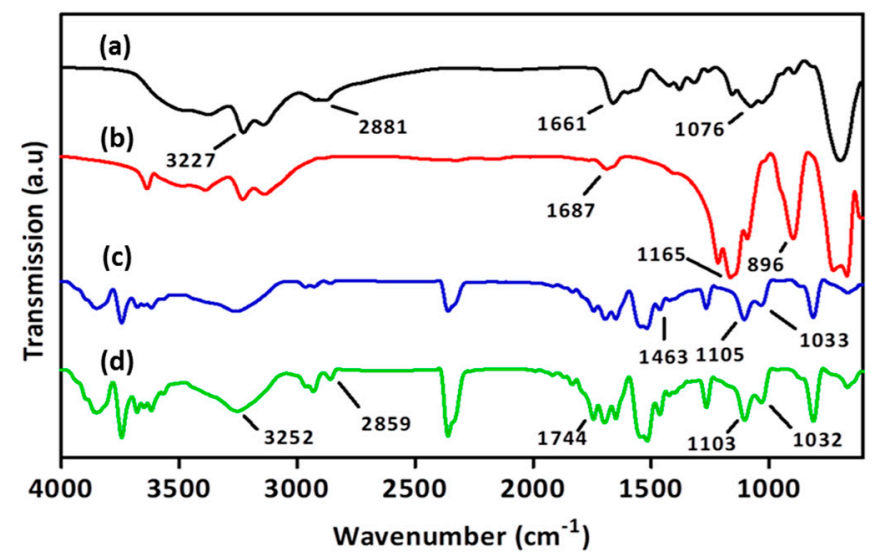

Figure 3. FT-IR spectra of (a) chitosan; (b) TPP; (c) FePC; and (d) FNP.

\subsection{Viability of C. tropicalis after Treatment with Antifungal Agents}

In order to test whether the $C$. tropicalis used in the experiment is resistant to fluconazole, the mostly commonly used antifungal medicine, the antifungal effect of fluconazole in the Candida species was carried out. The fluconazole concentration was $208 \mu \mathrm{M}$ or less. A non-drug resistant strain of C. albican was tested as a comparison. As shown in Figure 4a, dose-dependent inhibition was very pronounced in C. albicans. Comparatively, dose-dependence was very weak for C. tropicalis. Importantly, the inhibition of C. tropicalis was a lot weaker than the inhibition of $C$. albicans, especially at $208 \mu \mathrm{M}$. This indicates that $C$. tropicalis is strongly resistant to fluconazole.

As $C$. tropicalis has a strong resistance to fluconazole, other antifungal medicines are used in the clinic. Flucytosine, a well-known azole antifungal medicine, is one of the commonly used medications for $C$. tropicalis infections. Therefore, we tested the resistance of fluconazole-resistant $C$. tropicalis to flucytosine. The flucytosine concentration was $128 \mu \mathrm{M}$ or less. As shown in Figure $4 \mathrm{~b}$, a weak dose-dependence was shown after $24 \mathrm{~h}$ of treatment. The dose-dependence was slightly stronger in the $48 \mathrm{~h}$-treatment experiment. However, after the $24 \mathrm{~h}$ treatment, more than half of $C$. tropicalis survived at the maximum concentration of flucytosine. The cell viability was $\sim 40 \%$ after the $48 \mathrm{~h}$-treatment. This viability assay indicates that $C$. tropicalis is resistant to flucytosine.

(a)

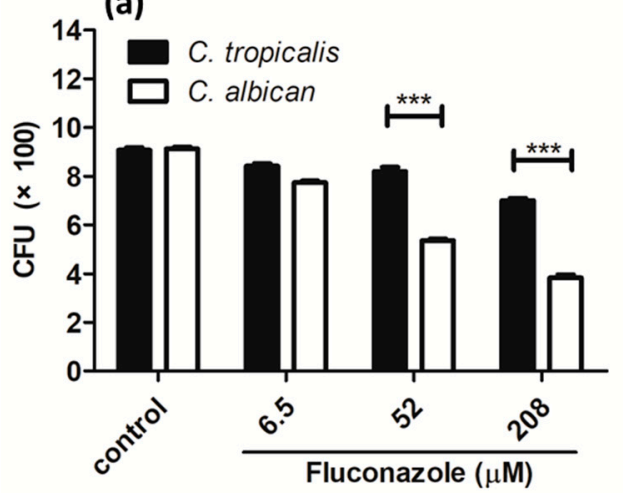

(b)

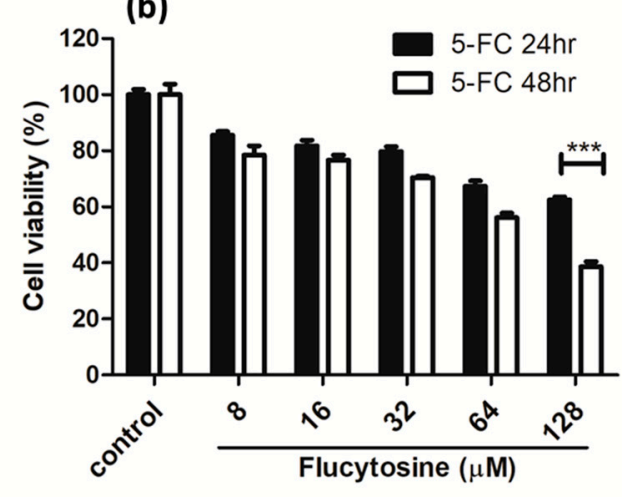

Figure 4. Viability of C. tropicalis after treatment with (a) fluconazole in planktonic culture in comparison to C. albican and (b) flucytosine (5-FC) in adherent culture. ${ }^{* * *} p<0.001$.

\subsection{PDT Effect on Planktonic and Adherent C. tropicalis}

Antimicrobial PDT using FNP against $C$. tropicalis was performed in both planktonic and adherent cultures. As shown in Figure 5a, in planktonic cultures, PDT with 40 and $80 \mu \mathrm{M}$ of FNP caused significant inhibition. This antimicrobial effect was much better than the effect achieved with antifungal medicines, such as fluconazole and flucytosine, which are shown in Figure 4. 
Candida species which develop biofilms are more drug-resistant and virulent [42]. Candida species develop biofilms when they are dispersed. Therefore, antimicrobial PDT using FNP was performed in adherent cultures of $C$. tropicalis. As shown in Figure $5 \mathrm{~b}$, cell viability decreased after performing PDT with FePC or FNP. The antimicrobial PDT effect was better when using FNP as compared to FePC. However, the antimicrobial PDT effect in adherent $C$. tropicalis was a lot weaker than in planktonic C. tropicalis. Consistent with our previous study on C. albicans [25], this result fulfills our prediction that it is more difficult to eradicate $C$. tropicalis when it is forming biofilms.

(a)

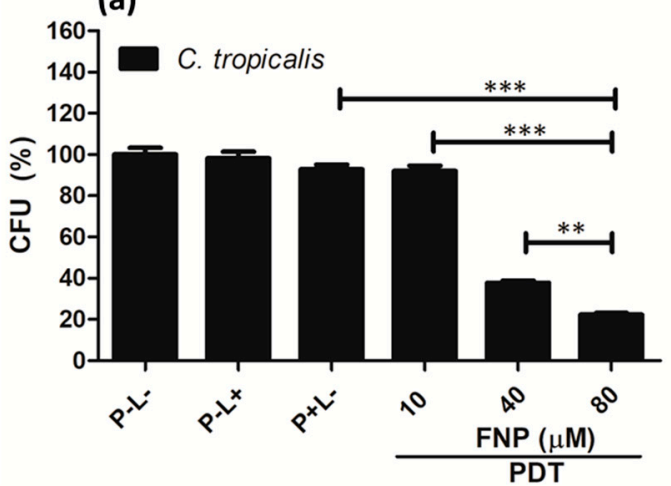

(b)

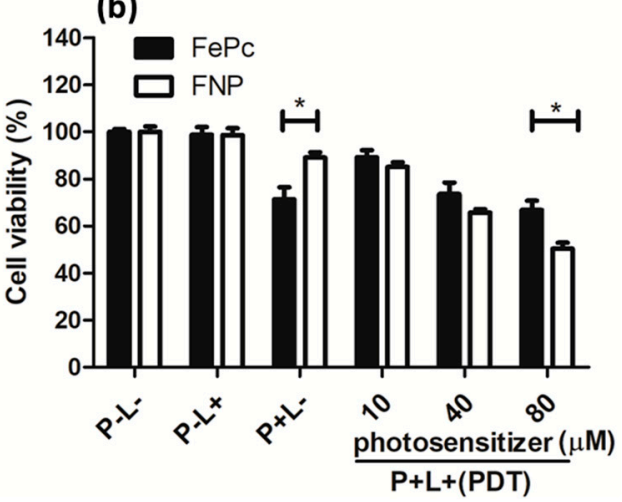

Figure 5. Viability of $C$. tropicalis in (a) planktonic cultures with FNP-PDT, and (b) adherent cultures after PDT with FePC or FNP. P and L represent photosensitizer and light, respectively. The symbol + indicates that the specific factor was used, and - indicates that the specific factor was not used. ${ }^{*} p<0.05,{ }^{* *} p<0.01$, or ${ }^{* * *} p<0.001$.

\subsection{The Effect of Combined Therapy using Flucytosine and FNP-PDT on C. albicans in Adherent Cultures}

To fully eradicate C. tropicalis, we combined traditional flucytosine therapy and FNP-PDT. The antimicrobial effect of this combination was also tested in different sequences. As shown in Figure 4, flucytosine $(128 \mu \mathrm{M})$ caused $40-60 \%$ cell death. As shown in Figure 6, the photosensitizer (FNP) had no effect on cell viability before illumination. After the flucytosine treatment, FNP-PDT largely decreased the viability of $C$. tropicalis cells in a dose-dependent manner. Cell viability was $\sim 25 \%$ when the treatment involved flucytosine and subsequent PDT with $80 \mu \mathrm{M}$ FNP. Alternatively, using FNP-PDT prior to the flucytosine therapy caused more significant cell death. The cell viability was only $~ 10 \%$ when PDT was used with $80 \mu \mathrm{M}$ FNP and flucytosine treatment sequentially.

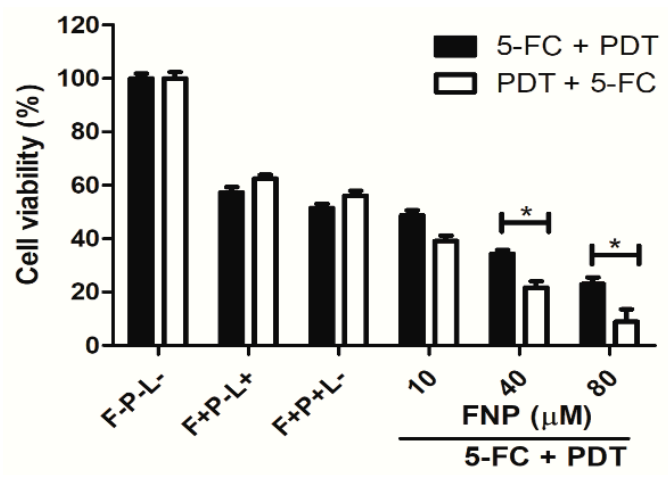

Figure 6. Viability of C. tropicalis in the combined therapy of flucytosine (5-FC) and FNP-PDT sequentially shown in the solid columns, and the reversed order shown in the unfilled columns. F, P and L represent flucytosine, photosensitizer and light, respectively. The symbol + indicates that the specific factor was used, and - indicates that the specific factor was not used. ${ }^{*} p<0.05$. 


\subsection{Cellular Morphology of C. tropicalis}

A unique feature of adherent $C$. tropicalis cells was their long pseudohyphae, as shown in Figure 7a. After FNP-PDT, the long pseudohyphae were greatly eradicated, as shown in Figure $7 \mathrm{~b}$. In contrast, flucytosine was toxic to yeast-like $C$. tropicalis. Flucytosine shortened the pseudohyphae but could not eliminate them, and the pseudohyphae were still widely present in Figure 7c. After the sequential treatment of flucytosine and FNP-PDT, as shown in Figure $7 \mathrm{~d}$, very few $C$. tropicalis cells were present, and the pseudohyphae were greatly eliminated. The eradication of pseudohyphae was more complete when FNP-PDT was performed prior to flucytosine treatment, as shown in Figure 7e,f. These images illustrate mostly yeast-like oval cells rather than long pseudohyphae. It is very clear that the $C$. tropicalis pseudohyphae and yeast-like cells were eradicated completely in this sequential FNP-PDT and flucytosine treatment.
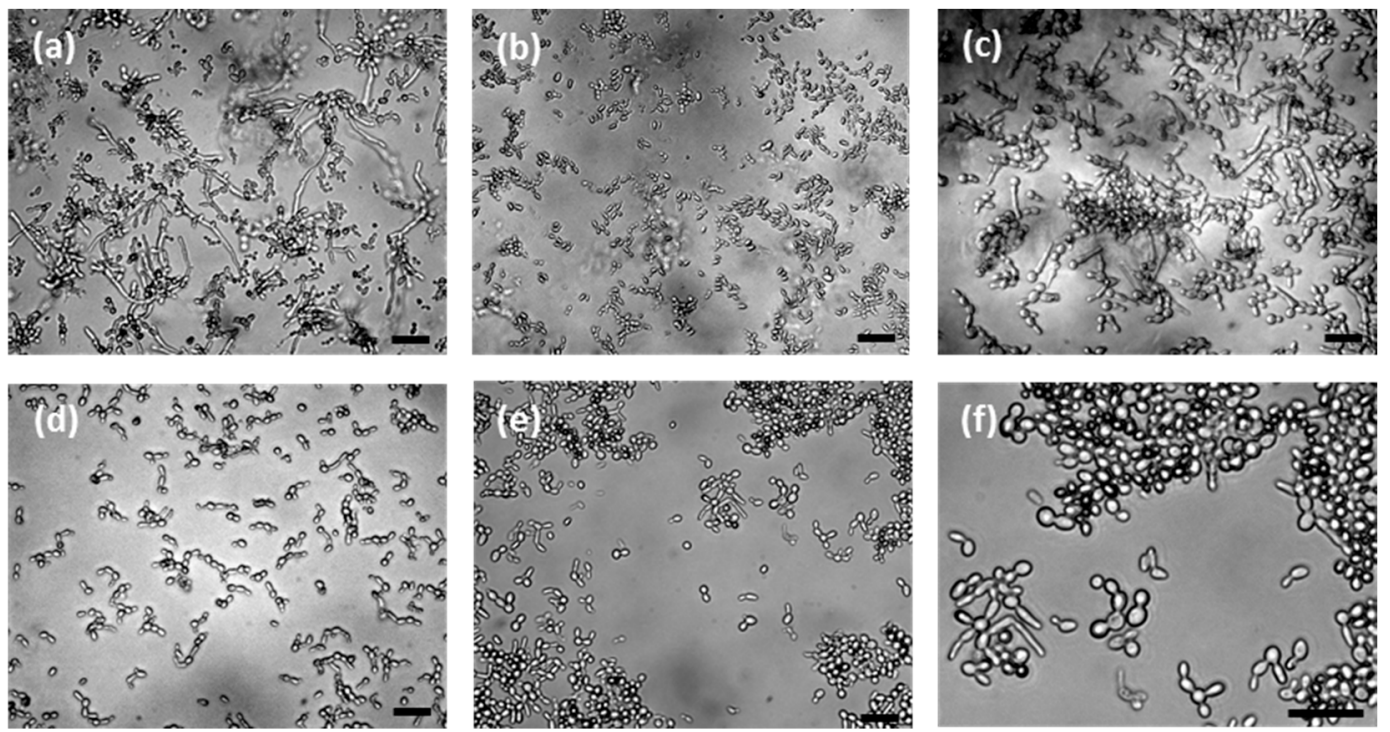

Figure 7. The morphology of C. tropicalis (a) before the treatment, and after treatment with (b) FNP-PDT; (c) flucytosine, and combination treatments of (d) flucytosine for $24 \mathrm{~h}$ followed by FNP-PDT, and (e) FNP-PDT followed by flucytosine treatment for $24 \mathrm{~h}$; (f) An enlarged image of (e). The scale bar represents $10 \mu \mathrm{m}$.

\section{Discussion}

Infection of $C$. tropicalis has become an important issue, especially for the strains resistant to traditional antifungal agents. The strain of $C$. tropicalis used in this study is resistant to fluconazole and flucytosine. Clinically, it is very difficult to treat infections caused by this strain. Therefore, alternative antimicrobial therapies are needed. As an alternative treatment, PDT is an efficient antitumor therapy. However, for antimicrobial therapies, the effect of PDT is limited, as has been previously reported $[25,43]$, and this is confirmed in this work. One major problem in antimicrobial PDT is the poor solubility and limited cell penetration of photosensitizers [44]. In this work, we encapsulated FePC with cationic chitosan/TPP nanoparticles. This approach solved the problem of solubility and provided a targeting effect through the charge interaction between the cationic chitosan/TPP nanoparticles and the anionic surface on the cell walls [45]. This encapsulation enhanced four-fold the availability of FePC to $C$. tropicalis cells. However, after the uptake enhancement using cationic chitosan/TPP nanoparticles, the uptake of FePC was only 5\%. This result strongly emphasized the importance of the drug delivery to microbes. How to greatly improve the cellular availability of therapeutics is a critical issue for all antimicrobial therapies.

In our study, FNP-PDT inhibited planktonic C. tropicalis very efficiently. However, FNP-PDT was not highly toxic against adherent $C$. tropicalis. As judged from the morphology, FNP-PDT 
abolished most pseudohyphae rather than killing the $C$. tropicalis cells. This finding on the effect of PDT on biofilms is consistent with previous studies on Candida species $[25,46,47]$ and Staphylococcus aureus [48]. In contrast to PDT, as an antifungal agent, flucytosine killed the $C$. tropicalis cells but could not eliminate the pseudohyphae efficiently. As the antibiotic resistance is mainly ascribed to the secretion of the extracellular matrix from hyphal and pseudohyphal organisms in biofilms [49], PDT and an antifungal treatment can be complementary. As proved in this study, the combined use of FNP-PDT and flucytosine greatly inhibits C. tropicalis replication. Interestingly, the sequence of these two treatments was critical to the viability of $C$. tropicalis. The use of FNP-PDT prior to flucytosine treatment provided better inhibition than the reverse sequence. The antifungal treatment of candidiasis usually takes months [50]. During the hours of flucytosine treatment, surviving C. tropicalis continued to develop pseudohyphae, weakening the antifungal effect. When the pseudohyphae were omnipresent, the subsequent FNP-PDT could only eliminate part of the pseudohyphae, resulting in the survival of $C$. tropicalis with long pseudohyphae. Therefore, the most efficient approach is to eradicate pseudohyphae by FNP-PDT prior to the treatment with antifungal agents to toxify C. tropicalis cells.

The resistance to antimicrobial agents is the most serious issue in antimicrobial therapy. After finding the great effect of PDT on biofilms of Candida species and bacteria, we need to further examine whether the PDT-treated strains overproduce drug-efflux proteins to remove photosensitizers.

\section{Conclusions}

The antibiotic resistance of Candida species is an important issue, and the development of alternative therapies to kill drug-resistant Candida cells and to reduce their biofilm-associated virulence is needed. Antifungal agents greatly eliminate the yeast form of $C$. tropicalis, but have little effect on pseudohyphae. Enhanced antimicrobial PDT using FePC-encapsulated chitosan/TPP nanoparticles can effectively eradicate pseudohyphae. The sequential use of FNP-PDT and flucytosine can strongly eliminate pseudohyphae, to reduce the virulence and inhibit the growth of fluconazole-resistant C. tropicalis cells. Potentially, this strategy can be applied to other drug-resistant pathogens.

Author Contributions: Y.-H.H. initiated experiments of C. tropicalis; W.-C.C., K.-H.Y. and C.-P.J. performed experiments; C.-I.L. led the project and wrote the manuscript.

Funding: This research was funded by Dalin Tzu Chi Hospital, Buddhist Tzu Chi Medical Foundation [DTCRD 106-E-05] and Ministry of Science and Technology [106-2113-M-194-009; 107-2113-M-194-005].

Conflicts of Interest: The authors declare no conflict of interest.

\section{References}

1. Ventola, C.L. The antibiotic resistance crisis: Part 1: Causes and threats. P T 2015, 40, 277-283. [PubMed]

2. Yapar, N. Epidemiology and risk factors for invasive candidiasis. Ther. Clin. Risk Manag. 2014, 10, 95-105. [CrossRef] [PubMed]

3. Sardi, J.C.; Scorzoni, L.; Bernardi, T.; Fusco-Almeida, A.M.; Mendes Giannini, M.J. Candida species: Current epidemiology, pathogenicity, biofilm formation, natural antifungal products and new therapeutic options. J. Med. Microbiol. 2013, 62, 10-24. [CrossRef] [PubMed]

4. Dimopoulos, G.; Karabinis, A.; Samonis, G.; Falagas, M.E. Candidemia in immunocompromised and immunocompetent critically ill patients: A prospective comparative study. Eur. J. Clin. Microbiol. 2007, 26, 377-384. [CrossRef] [PubMed]

5. Bennett, R.J. The parasexual lifestyle of Candida albicans. Curr. Opin. Microbiol. 2015, 28, 10-17. [CrossRef] [PubMed]

6. Fidel, P.L., Jr.; Vazquez, J.A.; Sobel, J.D. Candida glabrata: Review of epidemiology, pathogenesis, and clinical disease with comparison to C. albicans. Clin. Microbiol. Rev. 1999, 12, 80-96. [CrossRef] [PubMed]

7. Kothavade, R.J.; Kura, M.M.; Valand, A.G.; Panthaki, M.H. Candida tropicalis: Its prevalence, pathogenicity and increasing resistance to fluconazole. J. Med. Microbiol. 2010, 59, 873-880. [CrossRef] [PubMed]

8. Nobile, C.J.; Johnson, A.D. Candida albicans biofilms and human disease. Annu. Rev. Microbiol. 2015, 69, 71-92. [CrossRef] [PubMed] 
9. Whaley, S.G.; Rogers, P.D. Azole resistance in Candida glabrata. Curr. Infect. Dis. Rep. 2016. [CrossRef]

10. Chai, L.Y.; Denning, D.W.; Warn, P. Candida tropicalis in human disease. Crit. Rev. Microbiol. 2010, 36, $282-298$. [CrossRef] [PubMed]

11. Walsh, T.J.; Merz, W.G. Pathologic features in the human alimentary tract associated with invasiveness of Candida tropicalis. Am. J. Clin. Pathol. 1986, 85, 498-502. [CrossRef] [PubMed]

12. Wingard, J.R.; Merz, W.G.; Saral, R. Candida tropicalis: A major pathogen in immunocompromised patients. Ann. Intern. Med. 1979, 91, 539-543. [CrossRef] [PubMed]

13. Ding, C.H.; Wahab, A.A.; Muttaqillah, N.A.; Tzar, M.N. Prevalence of albicans and non-albicans candiduria in a Malaysian medical centre. J. Pak. Med. Assoc. 2014, 64, 1375-1379. [PubMed]

14. Tan, B.H.; Chakrabarti, A.; Li, R.Y.; Patel, A.K.; Watcharananan, S.P.; Liu, Z.; Chindamporn, A.; Tan, A.L.; Sun, P.L.; Wu, U.I.; et al. Incidence and species distribution of candidaemia in Asia: A laboratory-based surveillance study. Clin. Microbiol. Infect. 2015, 21, 946-953. [CrossRef] [PubMed]

15. Pappas, P.G.; Kauffman, C.A.; Andes, D.R.; Clancy, C.J.; Marr, K.A.; Ostrosky-Zeichner, L.; Reboli, A.C.; Schuster, M.G.; Vazquez, J.A.; Walsh, T.J.; et al. Clinical practice guideline for the management of candidiasis: 2016 update by the infectious diseases society of America. Clin. Infect. Dis. 2016. [CrossRef] [PubMed]

16. Pfaller, M.A.; Diekema, D.J. Epidemiology of invasive mycoses in North America. Crit. Rev. Microbiol. 2010. [CrossRef] [PubMed]

17. Odds, F.C.; Brown, A.J.; Gow, N.A. Antifungal agents: Mechanisms of action. Trends Microbiol. 2003, 11, 272-279. [CrossRef]

18. Sobel, J.D. Recurrent vulvovaginal candidiasis. Am. J. Obstet Gynecol. 2016, 214, 15-21. [CrossRef]

19. Kanafani, Z.A.; Perfect, J.R. Antimicrobial resistance: Resistance to antifungal agents: Mechanisms and clinical impact. Clin. Infect. Dis. 2008, 46, 120-128. [CrossRef]

20. Bondaryk, M.; Kurzatkowski, W.; Staniszewska, M. Antifungal agents commonly used in the superficial and mucosal candidiasis treatment: Mode of action and resistance development. Postepy Dermatol. Alergol. 2013, 30, 293-301. [CrossRef]

21. Thompson, D.S.; Carlisle, P.L.; Kadosh, D. Coevolution of morphology and virulence in Candida species. Eukaryot. Cell 2011, 10, 1173-1182. [CrossRef] [PubMed]

22. Agostinis, P.; Berg, K.; Cengel, K.A.; Foster, T.H.; Girotti, A.W.; Gollnick, S.O.; Hahn, S.M.; Hamblin, M.R.; Juzeniene, A.; Kessel, D.; et al. Photodynamic therapy of cancer: An update. CA Cancer J. Clin. 2011, 61, 250-281. [CrossRef] [PubMed]

23. Yin, R.; Agrawal, T.; Khan, U.; Gupta, G.K.; Rai, V.; Huang, Y.Y.; Hamblin, M.R. Antimicrobial photodynamic inactivation in nanomedicine: Small light strides against bad bugs. Nanomedicine 2015, 10, 2379-2404. [CrossRef] [PubMed]

24. Cieplik, F.; Deng, D.; Crielaard, W.; Buchalla, W.; Hellwig, E.; Al-Ahmad, A.; Maisch, T. Antimicrobial photodynamic therapy-What we know and what we don't. Crit. Rev. Microbiol. 2018, 44, 571-589. [CrossRef] [PubMed]

25. Hsieh, Y.H.; Zhang, J.H.; Chuang, W.C.; Yu, K.H.; Huang, X.B.; Lee, Y.C.; Lee, C.I. An in vitro study on the effect of combined treatment with photodynamic and chemical therapies on Candida albicans. Int. J. Mol. Sci. 2018, 19, 337. [CrossRef] [PubMed]

26. Allison, R.R.; Sibata, C.H. Oncologic photodynamic therapy photosensitizers: A clinical review. Photodiagn. Photodyn. Ther. 2010, 7, 61-75. [CrossRef] [PubMed]

27. Hamblin, M.R. Antimicrobial photodynamic inactivation: A bright new technique to kill resistant microbes. Curr. Opin. Microbiol. 2016, 33, 67-73. [CrossRef]

28. Gerber, D.E. Targeted therapies: A new generation of cancer treatments. Am. Fam. Physician 2008, 77, 311-319.

29. Shirasu, N.; Nam, S.O.; Kuroki, M. Tumor-targeted photodynamic therapy. Anticancer Res. 2013, 33, 2823-2831.

30. Sharma, S.K.; Dai, T.H.; Kharkwal, G.B.; Huang, Y.Y.; Huang, L.Y.; De Arce, V.J.B.; Tegos, G.P.; Hamblin, M.R. Drug discovery of antimicrobial photosensitizers using animal models. Curr. Pharm. Des. 2011, 17, 1303-1319. [CrossRef]

31. Lucky, S.S.; Soo, K.C.; Zhang, Y. Nanoparticles in photodynamic therapy. Chem. Rev. 2015, 115, 1990-2042. [CrossRef] [PubMed] 
32. Khor, E.; Lim, L.Y. Implantable applications of chitin and chitosan. Biomaterials 2003, 24, $2339-2349$. [CrossRef]

33. Ferreira, D.M.; Saga, Y.Y.; Aluicio-Sarduy, E.; Tedesco, A.C. Chitosan nanoparticles for melanoma cancer treatment by photodynamic therapy and electrochemotherapy using aminolevulinic acid derivatives. Curr. Med. Chem. 2013, 20, 1904-1911. [CrossRef] [PubMed]

34. Tsai, W.H.; Yu, K.H.; Huang, Y.C.; Lee, C.I. EGFR-targeted photodynamic therapy by curcumin-encapsulated chitosan/TPP nanoparticles. Int. J. Nanomed. 2018, 13, 903-916. [CrossRef] [PubMed]

35. Shrestha, A.; Kishen, A. Polycationic chitosan-conjugated photosensitizer for antibacterial photodynamic therapy. Photochem. Photobiol. 2012, 88, 577-583. [CrossRef]

36. Pati, F.; Adhikari, B.; Dhara, S. Development of chitosan-tripolyphosphate fibers through pH dependent ionotropic gelation. Carbohydr. Res. 2011, 346, 2582-2588. [CrossRef]

37. Bonnett, R. Photosensitizers of the porphyrin and phthalocyanine series for photodynamic therapy. Chem. Soc. Rev. 1995, 24, 19-33. [CrossRef]

38. CLSI. Performance Standards for Antifungal Susceptibility Testing of Yeasts, 1st ed.; CLSI Supplement M60; Clinical and Laboratory Standards Institute: Wayne, PA, USA, 2017; ISBN 1-56238-828-2.

39. Desnos-Ollivier, M.; Bretagne, S.; Bernede, C.; Robert, V.; Raoux, D.; Chachaty, E.; Forget, E.; Lacroix, C.; Dromer, F.; Yeasts, G. Clonal population of flucytosine-resistant Candida tropicalis from blood cultures, Paris, France. Emerg. Infect. Dis. 2008, 14, 557-565. [CrossRef]

40. CLSI. Reference Method for Broth Dilution Antifungal Susceptibility Testing of Yeasts; Third Infomational Supplement; CLSI Document M27-S3; Clinical and Laboratory Standards Institute: Wayne, PA, USA, 2008; ISBN 1-56238-667-0.

41. Bertoncello, P.; Peruffo, M. An investigation on the self-aggregation properties of sulfonated copper(II) phthalocyanine (CuTsPc) thin films. Colloids Surf. A 2008, 321, 106-112. [CrossRef]

42. Douglas, L.J. Candida biofilms and their role in infection. Trends Microbiol. 2003, 11, 30-36. [CrossRef]

43. Gad, F.; Zahra, T.; Hasan, T.; Hamblin, M.R. Effects of growth phase and extracellular slime on photodynamic inactivation of gram-positive pathogenic bacteria. Antimicrob. Agents Chemother. 2004, 48, 2173-2178. [CrossRef] [PubMed]

44. Sperandio, F.F.; Huang, Y.Y.; Hamblin, M.R. Antimicrobial photodynamic therapy to kill Gram-negative bacteria. Recent Pat. Antiinfect. Drug Discov. 2013, 8, 108-120. [CrossRef] [PubMed]

45. Lipke, P.N.; Ovalle, R. Cell wall architecture in yeast: New structure and new challenges. J. Bacteriol. 1998, 180, 3735-3740. [PubMed]

46. Dovigo, L.N.; Carmello, J.C.; Carvalho, M.T.; Mima, E.G.; Vergani, C.E.; Bagnato, V.S.; Pavarina, A.C. Photodynamic inactivation of clinical isolates of Candida using Photodithazine ${ }^{\circledR}$. Biofouling 2013, 29, 1057-1067. [CrossRef] [PubMed]

47. Marioni, J.; Bresoli-Obach, R.; Agut, M.; Comini, L.R.; Cabrera, J.L.; Paraje, M.G.; Nonell, S.; Nunez Montoya, S.C. On the mechanism of Candida tropicalis biofilm reduction by the combined action of naturally-occurring anthraquinones and blue light. PLoS ONE 2017, 12, e0181517. [CrossRef] [PubMed]

48. Kashef, N.; Akbarizare, M.; Kamrava, S.K. Effect of sub-lethal photodynamic inactivation on the antibiotic susceptibility and biofilm formation of clinical Staphylococcus aureus isolates. Photodiagn. Photodyn. Ther. 2013, 10, 368-373. [CrossRef] [PubMed]

49. Silva, S.; Henriques, M.; Martins, A.; Oliveira, R.; Williams, D.; Azeredo, J. Biofilms of non-Candida albicans Candida species: Quantification, structure and matrix composition. Med. Mycol. 2009, 47, 681-689. [CrossRef] [PubMed]

50. Pappas, P.G.; Rex, J.H.; Sobel, J.D.; Filler, S.G.; Dismukes, W.E.; Walsh, T.J.; Edwards, J.E. Guidelines for treatment of candidiasis. Clin. Infect. Dis. 2004, 38, 161-189. [CrossRef] [PubMed]

(C) 2019 by the authors. Licensee MDPI, Basel, Switzerland. This article is an open access article distributed under the terms and conditions of the Creative Commons Attribution (CC BY) license (http://creativecommons.org/licenses/by/4.0/). 